3. That, owing to a possible mistake in diagnosis, isolation in all cases should be imperative, until a reliable bacteriological examination can be made.

\title{
THE CONNECTION OF THE FEMALE GENERATIVE ORGANS AND LARYNGEAL AFFECTIONS.
}

\author{
By Seymotr Oppenheimer, M.D., of New York City,
}

Attending Laryngologist to Bellevue Hospital, O.D.P. ; Attending Laryngologist to the University Medical College Dispensary ; Senior Assistant to the Chair of Laryngology, University Medical College.

THE knowledge that some connection existed between the sexual apparatus and the vocal organs in males was known in remote antiquity among the Orientals, from the change observed in the character of the voice of the then familiar household servant, the eunuch. As the human mind progressed through various ages until the desire for music had become a prominent feature of Eastern life, attention was riveted on the harmonious voice of the young eunuch, and the practice of castration was made the means of livelihood in preparing boys for the church. Even until modern times the Italian castrated boy sopranos were of world-wide fame. F. V. Stein (1) has described a large and prosperous sect in Russia, known as Skopzi, and characterized by requiring castration of all its male members. After this sect had existed for some time as a secret society, attention was called to it by the change in the voice of its members.

In the lower animals the influence exerted by the sexual organs over the laryngeal mechanism is well shown by the stag roaring for its mate during the rutting period, but yet never producing laryngeal sounds at other times; this is also observed in birds making increased vocal efforts during the breeding season, and in the singing birds the rhythm and flow of song is more beautiful during their sexual periods than at any other time.

In a few observed cases of male singers of mature age where castration had been performed, or the generative organs were destroyed by disease, marked changes were noted, not only of the speaking, but especially of the singing voice. At the age of puberty the larynx grows rapidly, and the voice of a boy "breaks" in consequence of the lengthening of the cords, generally falling an entire octave in pitch; a similar change, but much less in amount, occurs at the same period in the female. In many individuals coughing ean be excited by stimulation of distant 
sensory nerves, as from the uterus, mamma, and ovaries. (2) From these observations it can readily be seen that some connection, be it what it may, exists between the generative organs and the larynx, not only in the male, but also in the female sex. A strictly scientific explanation of these phenomena, both in health and disease, is not always discoverable, but that such an intimacy does exist may be accepted. The extreme delicacy of the mechanism of the larynx as concerned in phonation and vocalization renders it reflexly susceptible to slight changes in other portions of the organism, the proper and accurate adjustment of the vocal cords necessary to the singing voice requiring an elaborate and perfect nerve control. As will be seen later on, we must have perfect innervation of the laryngeal apparatus, or its usefulness will be seriously impaired.

Following out this line of reasoning, and studying the nervous mechanism of the vocal organs, and then observing the intimate connection of the sympathetic nerves with the female generative organs, and our speciality as to the influence exerted by one or the other will with some scientific accuracy be permissible. Anatomically the female larynx differs considerably from that of the male in being only about one-half as long and yet very nearly as wide as the male vocal organ ; the rima glottidis is also one-third shorter in the female. The natural position of the larynx is on a plane considerably higher in the female; this disparity in shape, size, and position accounts for the difference in the register of the voice between the two sexes.

The relation existing between the two organs may be either through the bloodvessel connection or by way of the nervous aplaratus, the sympathetic and vaso-motor nerves principally acting in both instances. Let us take as a hypothetical case a woman with a moderate degree of eatarrhal laryngitis of some years' standing, from some cause not here necessary to specify: uterine congestion occurs, a few hours later there is aphonia. The question naturally arises, as to the relation between the two affections, Is the aphonia due to the pre-existing laryngitis, or is it dependent upon the uterine congestion? Local treatment of the larynx is without avail, but immediately after the proper treatment directed to the uterus has been applied, the aphonia disappears, to return only when the pelvic organs again depart from the normal. A prejudiced mind only would hesitate in ascribing some relation to cause and effect between the larynx and uterus. In what way or manner is this correlation of sexual and vocal organs brought about? A study of the nervous connection between the two organs 
will greatly aid in elucidating the problem. The irritation from the diseased uterus is first transmitted through that portion of the pelvic sympathetic system known as the inferior hypogastric plexus, being in intimate association with the female pelvic organs. From this plexus we find the reflex action transmitted to the solar plexus; intimately associated with this group of nerve tissue are fibres of the pneumogastric nerve. As the motor fibres of the pneumogastric nerve are derived in great part from the spinal accessory, and these fibres are in direct relation with the larynx through its motor supply, the inferior or recurrent laryngeal, it becomes apparent through what nervous mechanism the uterine irritation is expressed by various laryngeal manifestations.

That this relation of the spinal accessory or recurrent laryngeal motor fibres to the larynx exists is shown by experimental section of either of these two nerves, with consequent complete loss of voice. Pressure by neighbouring morbid growths to the point of complete isolation of these fibres produces the same results. Among the fibres of the sympathetic are found branches going to the oesophagus, thyroid gland, and larynx, controlling and regulating the tonus of the bloodvessel walls; these are the so-called vaso-motor nerves. These fibres are at the upper portion of the great sympathetic system, and at its inferior portion we also find special vaso-motor fibres supplying the vagina, uterus, and ovaries. Stimulation of all these nerve channels causes increased activity of the organs (i.e., uterus and larynx) supplied by them, and at the same time there is produced contraction of the vessel walls, resulting in a diminished supply of blood. Section of these nerves is followed by dilatation of the bloodvessels, with subsequent derangement of the circulation, and ultimately of the nutrition. The morbid nerve impulse, originating from the uterus or ovaries, may therefore produce changes in the larynx in two ways: either by reflex inhibition of the inferior laryngeal through the vagus and sympathetic, or by irritation of the vaso-motor apparatus, with resultant disturbance of the laryngeal blood-supply, as seen above. Probably, in the majority of cases, both these means are factors in the production, not only of the voice alterations, but in the changes seen in the mucous membrane, such as anæmia or congestion.

The alterations as revealed by the laryngoscope may be those seen in the various forms of catarrhal inflammation incident to this organ, or various paralyses or spasms of the cords may also be observed. In a number of cases, however, nothing will be revealed on examination, as the following case will show: 
Madam C. S., aged twenty-seven years, a professional singer, soprano, married, became pregnant. Came to my office complaining of complete aphonia. She stated that the loss of voice came on ten weeks after conception, and has remained so ever since. Examination of the nares, fauces, pharynx, and larynx revealed nothing, the latter organ to all appearances being normal. Three weeks after the birth of her child the voice suddenly returned, and is now more beautiful than ever, and has continued so since.

Seiler (3), who has given a great deal of attention to this somewhat obscure subject, says that he has observed a peculiar condition of the mucous membrane of the upper air passages, which is neither an anæmia nor a congestion and yet is abnormal, and this pathological condition is found to be invariably caused by morbid changes in the female pelvic organs. So certain and distinct is the evidence of uterine disease in the upper air passages that the laryngologist can diagnose the presence of uterine trouble merely by the inspection of the pharynx and larynx. To conclusively prove that the diagnosis of uterine disease could be made with the laryngoscope, Dr. Seiler, in conjunction with Professor Howard A. Kelly, made a series of investigations: the larynx was examined and the diagnosis of uterine disease was made. Without seeing the diagnosis already made, Professor Kelly made a vaginal examination of the cases, and in every instance confirmed the views of $\mathrm{Dr}$. Seiler. He further says: "By slight differences in the appearance of the mucous membrane and in the position of this pathological condition of the upper air passages, a distinction can even be made between uterine and peri-uterine disease, and I have found that when the condition referred to is more prominent in the larynx and pharynx, the case is intra-uterine." This condition of the larynx, so ably described by Seiler, consists in a peculiar bluish-red tint of the mucous membrane, compared to the atmosphere of a clear sunset in the fall of the year. The membrane itself appears relaxed, and here and there over the surface there is enlargement of the follicles with a "muddy" appearance of the vocal cords, which are also relaxed and apt to flutter during the production of low notes; the secretions are but slightly increased in amount. The subjective symptoms are a slight hacking laryngeal cough, little or no expectoration, and a feeling of heat and burning in the throat, and in many cases a feeling of choking coming on at irregular intervals usually from excessive mental or physical exertion.

The second case illustrating this subject came to my office complaining of metrorrhagia and hoarseness. Patient was eighteen years of age. Had had numerous severe hæmorrhages from the 
uterus, these hæmorrhages being invariably followed by hoarseness. Examination of the larynx showed extravasation of blood into both vocal cords. The local condition was not treated, but constitutional remedies were given for the anæmia present, and after eight weeks the extravasations gradually disappeared and the voice became normal, remaining so until the present time, now a lapse of two years. Patient has not had any hæmorrhages during this period. Is now married and pregnant.

During the various changes incident to the female life certain peculiarities appear as affecting the larynx reflexly from the generative organs, both from the ovaries and uterus, but especially from the latter organ. The changes observed in the larynx will be taken up in the following order: Larynx at puberty, during adult life, at menstruation, at the menopause, and during pregnancy.

Puberty.-At the stage of puberty in the female, as before seen, there is increased growth of the larynx, with certain modifications in the voice, already mentioned. The most frequent affection that occurs as the result of the rapid developmental changes in the generative organs is spasm of the glottis (4). This occurs as one of the protean forms of hysteria, and females at the age of puberty seem especially liable to transient glottic spasms. At this time we also see sudden congestion of the larynx, especially before the appearance of the first menstrual discharge. The excessive flow of blood to the vocal organs is but temporary, and is due to the unstable condition of the vaso-motor system at this critical period in the life of the young girl. If the larynx be examined, it will be seen to be excessively red in colour, with the vessels prominent over all parts except the vocal cords, which remain unaffected; the aryepiglottic folds and interarytenoid space are coloured a deep red, and the epiglottis is similarly affected. This may occur several times during the day, and lasts but a few minutes, disappearing as rapidly as it came. If the congestion continues for any length of time, cdema of the glottis will result, often requiring active measures for the relief of the dyspnœa.

Adult Life.-Under the caption of changes seen in the female larynx during adult life are included all the conditions the result of uterine disease not occurring during any particular epoch as menstruation, the menopause, etc. Many of these laryngeal affections have been ascribed to hysteria, but careful study of the case has later developed the genital tract as being active in the production of the laryngeal disorder. During the female adult life many varieties of pelvic disease manifest themselves. In a certain number of these cases the larynx becomes affected in various ways as a 
result of the pelvic disorder. For the sake of systematic study we may divide the laryngeal affections into two general classes: those affecting the voice without observable changes in the larynx, and, secondly, definite morbid alterations of the laryngeal structures, readily seen by laryngoscopical examination, and remaining unchanged in spite of direct treatment applied to this region, but rapidly disappearing on removal of the uterine or ovarian irritation. In the majority are the so-called hysterias affecting the larynx without appreciable lesions. The following case, observed by S. Johnson Taylor (5), will illustrate this form of reflex action:

A female suffered from hysterical aphonia for thirteen years; various forms of laryngeal and constitutional treatment were used, but had no effect on the loss of voice. Examination of the larynx gave no evidence of disease. At the expiration of this time an ovarian cyst was diagnosed and removed, and immediately after the operation the voice returned to its normal condition.

The relation of cause and effect is beautifully shown in this case; the protean manifestations of uterine or ovarian irritation usually concern the laryngeal motor apparatus, the sensory nerves not being affected in many instances, although pain referred to the region of the epiglottis, or even to the interior of the larynx, may occur as the result of endometritis and misplacements of the uterus, this latter condition, and especially a bad grade of retroflexion, being in my experience quite a common cause of cough. The cough in this instance is irritative, as if a foreign body were present in the larynx, and resembles to a great extent that produced by hypertrophy of the lingual tonsil. It comes on frequently, not in attacks, but is quite constant during the day, and disappears at night, and is short and sharp, and not productive of expectoration. Examination of the upper respiratory tract, and especially the larynx, will show nothing except in long-standing cases, where from the constant expiratory blast a moderate degree of passive congestion has taken place. Replacing the uterus in its proper anatomical position causes an almost miraculous disappearance of the cough, and the laryngeal congestion rapidly subsides.

The paralyses of the intrinsic muscles concerned in vocalization may be so varied, and at the same time coincident with spasm of some of the other muscles, that a diagnosis is somewhat difficult. This is especially true when the cause lies in the generative aplaratus, as one muscle alone may be affected or an entire group. Adductor paralysis is probably the most common form, as seen in hysteria dependent upon disorders incident to the female. Norell Mackenzie (6) reports one case-a spinster, aged thirty-seven years, 
suffering from aphonia of two years' standing. Previous to this, she had for some time been treated for the uterine disease; she was very weak, but free from organic disease. On vocalization, the cords were approximated properly in the anterior three-fourths of the glottis, but remained widely open in the posterior fourth, leaving a triangular area of separation. Various remedies were tried, both constitutional and anti-hysteric, and endo-laryngeal faradism was employed, but no sounds could be elicited. The case was then anæsthetized, but on recovering consciousness the voice did not return. Although this case was under his observation for twelve years, the voice was never restored.

I think it is agreed by the majority of observers that the uterus is more often at fault than its appendages. In singers imperfect voice is seen much more frequently from uterine disease than from ovarian, although, of course, the ovaries may in some cases of aphonia and irritation of the mucous membrane of the larynx play the allimportant part as the etiological factor. It is quite common in hysterical subjects to find areas of anæsthesia, parasthesia, and especially hyperæsthesia, situated over various organs, the ovarian regions being especially the site of these perverted or sensitive areas. In other cases pressure over one or both ovaries may elicit exquisite sensitiveness without pain in other portions of the body; in a few cases pressure in this region will elicit pain elsewhere, and, as intimately connected with our subject, the distant area of perverted sensation may be the larynx. Trifilette, of Naples (7), reports a case of hysterical aphonia in which there was a hysterogenic zone over the left ovarian region; pressure on this area would invariably produce a lowering of the voice. As in the majority of these cases, the larynx was normal. Changes in the singing-voice, and less rarely in the speaking-voice, from disease of the generative apparatus are comparatively frequent, especially in professional singers; $;$ and, as will be seen later on, this voice alteration occurs usually during menstruation, but it may occur at any time, especially if much strain has been put upon the vocal organs. As has been seen, the nervous mechanism governing the movements of the vocal cords must be in perfect order to produce the best results. A slight disturbance will affect the number of cord vibrations necessary to produce a given note, and it is often the cause of much astonishment that laryngeal disturbances are not more often produced.

In the second class of cases occurring under this heading, we find well-defined lesions of the laryngeal mucous membrane resulting from the uterine diseases. Congestion is most frequent, varying in degree from a mere furitive blushing of the mucosa to intense 
blood stasis, causing the entire interior of the larynx to become deepred in colour, and if continued for any length of time ultimately crossing the border-line, and developing into a subacute or chronic laryngitis. The degree of congestion present in the larynx as a general rule bears no relation to the amount of disease present in the uterus. A mild catarrhal endometritis may be the cause of an intense form of laryngeal inflammation, while severe inflammation of the endometrium with stenosis of the cervical canal will be productive of but a slight cough, with possibly little or no congestion or changes of any kind observable in the larynx. Anæmia of the larynx may be the principal sign of involvement from uterine changes, especially if a long-continued metrorrhagia has existed. Spasm of the glottis, or of individual laryngeal muscles, may be seen, due to a uterine fibroid; as, like that mentioned previously in regard to congestion, the degree of spasm bears no relation to the size of the uterine growth. C. H. Leonard (8) reported the case of a young woman, a professional singer, with anteflexion and narrowing of the uterine canal with severe endometritis. No changes were olserved in the larynx, but there was a loss of the purity of her tones and lessening of the range; from being a high mezzo-singer she could only sing contralto. After appropriate uterine treatment, with no laryngeal medication whatsoever, she not only recovered the singing-voice, but gained in addition two full notes.

It is difficult at times to realize why the larynx should become affected, and yet present no macroscopic change. That there are some microscopic lesions of the deeper laryngeal tissues or changes in the nerves seems necessary, to allow of the loss of phonation or change in the character of the singing-voice. In all these cases there are probably laryngeal changes, which, although not perceptille to the eye, yet render the organ susceptible to the least disturbance of nerve origin elsewhere in the body. In singers, from the constant use of the vocal organ, and often after its improper use, especially in the actress, where occasion has demanded a change of voice other than usually used, some bad results must be seen ; and it is chiefly in this class of patients that we observe the effects in the larynx directly caused by some weakness in that organ, already inherent, but remaining latent possibly for a considerable period of time, and is kindled into a flame by the uterine disease.

The Menstrual I'eriod.-The phenomena presented by the larynx during the menstrual period will to a great extent depend upon two factors: whether the generative organs are healthy or diseased at the time of the monthly flow. This period of the 
female life seems prolific in producing laryngeal disorders. During the first few periods of menstruation a group of laryngeal symptoms are sometimes observed, and known as " chorea laryngis" or laryngeal vertigo. There is a momentary spasm of the glottis, preceded by a tickling sensation in the throat, expiration of air is arrested for a moment, then a loud cough relaxes the spasm. This is frequently followed by insensibility for a few minutes, but no stupor results. The attacks may come during sleep, and are characterized by spasms of the laryngeal muscles, the inco-ordination of muscular movements produced resulting in a series of peculiar sounds resembling the bark of a dog. A nervous laryngeal cough is sometimes an accompaniment of the menstrual period, being most marked at the beginning and termination of the flow, and in the interval practically being held in abeyance. Certain changes in the voice are frequent during this period in some cases; the voice may be husky, resembling the masculine voice very much, and changing from day to day, dependent apparently upon the quantity of the menstrual flow.

Of more importance and more often seen, and but too frequently neglected by the laryngologist, are the changes in the singing-voice during the menses; these alterations are as numerous and varied as there are women affected, each case being a study of itself. The gravity, force, and timbre of the voice are defective, and so well known is this effect of menstruation upon the singing-roice that many prominent vocalists rest their larynx at this time, or, if absolutely necessary to sing, will use the vocal organs as little as possible. If the voice is used in one in whom the uterine congestion affects its quality at the regular monthly periods, we shall find on examination of the cords that they are slightly congested, while the interior of the larynx will be redder than normal. Should excessive use be made of the voice at this period in a susceptible female, an acute laryngitis is very apt to result, differing in no way from the ordinary laryngitis except in its etiology and the violence of the inflammation, ameliorating on the cessation of the menses, but leaving a moderate degree of hyperæmia and inability to vocalize for a few days afterwards. From the same cause hæmorrhage into the vocal cords may result, as in the case reported by Poyet (9) of an opera-singer obliged to strain her voice during the menstrual period. There was a submucous hæmorrhage of both vocal bands; this occurred several times in the same situation and from the same cause. Instead of being confined to the cords, the extravasation of blood may be distributed over various parts of the laryux, constituting a typical hamorrhagic laryngitis, as seen in the 
following case : A female, aged thirty; during a menstrual period, without any extraneous causes, the symptoms of laryngitis appeared. The case was seen by Treitel (10), who, on laryngoscopic examination, found extravasations of blood at various points of the laryngeal mucosa.

When the generative organs are the seat of pathological changes reflex manifestations are occasionally seen in the larynx only at the menstrual period, or, if amenorrhcea exists, the affection of the upper respiratory tract will become evident at the time during which the flow should have been present. In shop-girls, who are compelled to stand for many hours at a time, we often find them subject to a nervous laryngeal cough ; invariably associated with this cough we find dysmenorrhoea and frequently a mild grade of orarian irritation. One case seen several years ago, of which the notes are not now available, required the injection of morphia to relieve the intense pain from which she suffered; dysmenorrhœa was present at every period, and accompanying it was a sharp, nerrous cough, disappearing in the interim. As further illustrating this condition, may be cited the case seen by De la Sota, of Seville (11): A young lady, aged twenty-five years, somewhat hysterical, had suffered for two years from uterine disorders. During treatment for this condition copious menstruation occurred; this was followed by a complete suppression for two months, and at the expiration of that time, coincident with the menstrual period, there was an attack of hæmorrhage from the larynx and laryngo-pharyngeal catarrh.

Various paralyses of the laryngeal muscles may occur during the menstrual flow, being identical in character with those observed as the result of other conditions. As in the majority of cases when the element of hysteria is present, the adductor group of intrinsic muscles are the ones usually involved. Mackenzie cites a case due to amenorrhcea. This form of paralysis is very common in dysmenorrhœa and other menstrual disorders, especially in single women between the age of thirty and forty years. Compaired (12) reports an extremely interesting case of a young girl, twenty-one years of age. She had not menstruated for one year, but, replacing it at each period, she suffered an acute attack of laryngitis. Previous to this her menses were normal, and there were no laryngeal troubles. At the time of observation, in addition to the laryngitis and amenorrhœea, there was chlorosis, exophthalmic goitre, and tachycardia. In some respects this case calls to mind the so-called vicarious menstruation, in which peculiar condition epistaxis or laryngeal hæmorrhage usually takes place at the monthly periods 
of the menstrual flow; but in a few cases cardiac pains, frontal headache, or, as seen in this case, laryngitis may be the alternate.

The Climacteric.-During the climacteric period the pelvic organs undergo a transitional stage, and are unusually susceptible to pathological changes. At this time reflex changes are common in various parts of the nervous system, and occasionally the larynx is more particularly the seat of the reflex action. Semon (13) has contributed to medical literature his experiences of the "sensory throat neuroses" seen at this period, and, as it contains many valuable suggestions, will be quoted more or less extensively. In all these cases a neurotic or hysterical element is prominent. The sensations referred to by the patients may be of two kinds, either parasthesias or neuralgias; but these sensations differ in each case, being described as if a foreign body was present, or burning, scratching, dryness, soreness, tickling, and, in fact, nearly all the forms of perverted sensations are described, so that the list may be extended in an indefinite manner. Some of these sensations, such as the dryness, as claimed by this author to be due to the "change of life," are very probably the result of pre-existing pharyngeal catarrh of the atrophic form, the sensation of intense dryness, as seen in this disease, often being referred to the laryngeal region.

The location of an abnormal throat sensation is most difficult; it frequently happens in my experience that a foreign body, such as a minute bristle or fish-bone, located in one of the crypts of the faucial tonsil will be described by the patient as being in the larynx, or vice versâ. It is the same way with these parasthesias at the menopause, the patient with great difficulty locating the area of perverted sensation.

The neuralgic pains are more frequent in the pharynx, radiating to the ears, and located in a definite area; but more rarely they are complained of as being laryngeal, and then seem to vary in location, constantly shifting from one side to the other, or remaining in one part of the larynx for twenty-four hours and then changing to another part, the mere protean character being a valuable aid in the diagnosis. The intensity of the laryngeal sensations vary greatly, in the majority of cases being merely uncomfortable, but in a few extremely neurotic individuals intense pain may be complained of. This condition occurs usually in the better class of patients, and especially among educated women. General bodily depression may be well marked, and if the pain is long continued and severe, the early signs of melancholia may be observed. These throat symptoms may precede or accompany the menopause, in some cases being the first and only sign of the 
impending sexual changes, or, in an equally large number of cases, will be associated with the other phenomena accompanying the climacteric period. The objective symptoms are not in any way characteristic; in this class of cases, when the nervous throat disorders are the result and part of the change of life, nothing will be seen on laryngeal examination to give any clue to the distress complained of. Of course, in the majority of all women who have reached the age of the menopause, throat changes are very common, an absolutely normal throat being a rarity, so that we will often find some atrophy of the oro- or laryngo-pharyngeal tissues, or slight enlargement of the lingual tonsil. As the larynx is not so often affected as is the pharynx, we will rarely find any conspicuous changes.

These neuroses disappear on the cessation of all the phenomena of the menopause, but judgment must be exercised in making known the prognosis, as in many cases the changes may last for two or more years, the laryngeal symptoms continuing until the organism has settled into its new conditions. Care must also be taken, in making the diagnosis of this condition, that due credit be given to any pathological changes in the larynx that may exist at the time of the menopause, and possibly be the cause of the parasthesia, etc. Because a woman at this period complains of various neuroses of the larynx, it does not always signify that the generative organs are the founs et origo of the trouble; the diagnosis can only be made by eliminating other factors as causative agents.

As we have seen in other periods of the female existence, inflammations of the laryngeal mucosa may be present from changes in the sexual apparatus as well as pure nervous changes. This applies equally well to the menopause. All grades of inflammatory laryngeal troubles may be seen, but a mild form of laryngitis, lasting but a few days, then repeated at frequent intervals, is most common. In a large number of cases a mild grade of previously existing catarrhal laryngitis becomes more marked, while in a few cases a subacute form of catarrhal laryngitis with little tissue change continues during the entire climacteric period. The voice in these cases is rarely affected, although it may be slightly husky in the morning, but immediately becomes clear after removal of a small particle of mucus which has collected in the larynx during the night. Glasgow (14) reports six cases of spasm of the glottis incident to the menopause; in four there were catarrhal laryngitis, in one a malignant uterine tumour, and in the sixth no cause of the laryngeal cedema was ascertainable except the period of life. In the last case the attacks lasted for two years; 
there had been an entire cessation of menstruation, and after a profuse uterine hæmorrhage they entirely disappeared.

Pregnancy.-During pregnancy the larynx, like many other parts of the body, may participate in reflex actions due to the changes taking place in the generative organs. Laryngeal reflexes in comparison with the other irregular symptoms incident to the pregnant state are quite rare. The case of aphonia as previously described was a typical example of uterine reflex action from this condition. An irritative cough is occasionally observed in pregnant women, in some cases so severe as to alarm the patients; no changes are observed in the upper respiratory tract, and, despite medication, it continues until the woman is delivered, when it immediately ceases. In other cases the cough may be but slight, replacing the familiar morning sickness; after arising in the morning there will be a severe spell of coughing, without expectoration, lasting for several minutes, then it will not be present again until the next day at the same time. In a case quoted by Lennox Browne of nervous laryngeal cough due to pregnancy, it always occurred in the early days of conception, and invariably at the period of "quickening." This woman had several children, and the first symptoms of pregnancy was the cough; she regarded it as pregnancy even before other circumstances confirmed her state.

Spasm of the glottis and inflammation of the larynx may also accompany the pregnant state, grave morbid alterations of the laryngeal structures being rare at this time; but a unique case reported by Bayer (15) merits a somewhat detailed description. His case was a female, aged thirty-four years, complaining of pronounced symptoms of laryngeal obstruction, with dyspnoea, cough, aphonia, and expectoration. Laryngeal examination revealed an ulcerative laryngeal tuberculosis, with tumefaction of the epiglottis, arytenoids, and inflammation of the larynx. The glottic opening had become so reduced in size from the swelling that tracheotomy seemed indicated at short notice. A short time after this the woman, who was three months pregnant, aborted and had several uterine hæmorrhages; eight days later the tumefaction of the larynx had so diminished under the severe hæmorrhages occurring as the result of the abortion that tracheotomy was no longer indicated. In commenting on this case, he makes the statement that one must never neglect to ascertain the condition of the generative organs in females suffering from important laryngeal disorders.

After operations on the uterus or ovaries, no pathological laryngeal changes occur, as far as ascertainable from the literature on the subject. The singing-voice is sometimes changed after 
removal of the ovaries, but as far as the talking-voice is concerned, but little alteration can be observed in the majority of cases, while in a few women the voice seems to became coarser in the lapse of years; that this has any relation to the oophorectomy is somewhat doubtful. I have studied the speaking-voice (the girl could not $\operatorname{sing}$ ) in one case of a young woman, eighteen years of age, who hal had her ovaries removed; and now, after a lapse of five years, no rocal changes have appeared. As far as the singing-voice is concerned, it seems that some alterations in its character take place in a limited number of females after removal of both ovaries. Castex (16) carefully studied the voice in six women, under thirtyfive years of age, who had been subjected to öophorectomy. In one instance the effect of the operation was damaging, the voice became harsh, especially in the high notes, and unfitted her for singing. In another case, that of a mezzo-soprano, four low tones were added to the compass of the voice without any change in its strength or timbre. In the remaining cases, either there were no changes in the voice, or whatever alterations there were could not with certainty be attributed to the operation. Castex believes that the chance of damage to the voice from oophorectomy is too small to count as a contra-indication to the operation. After hysterectomy, no observable alterations of the voice take place.

The study (17) of the relations between the larynx and sexual apparatus opens up a new field of research of pleasing landscape and almost boundless horizon, which bids to its exploration not only the physiologist and pathologist, but also the biologist.

Above all, it brings us face to face with a serious problem of life, an interesting enigma whose significance it will be the task of the future to divine.

\section{ReFERENCES.}

(1) F. v. Stein. "Zeitsehrift für Ethnologie," 1875.

(2) Landois and Stirling, "Text-book of Physiology."

(3) Seiler, "Diseases of Nose and Throat."

(4) Lennox Browne, "Diseases of the Throat."

(5) S. Johnson Taylor, “" British Medical Journal," November, 1894.

(6) Morell Mackenzie, "I)iseases of the Throat and Nose."

(7) Trifilette, "Archives di Laringologie," 'Turin, July, 1895.

(8) C. H. Ieonard, "Journal of the American Medical Association," July 9th. 1892.

(9) Poyet, "Revue de Laryngologie," Paris, September, 1894.

(10) Treitel, "Deutsche Medicinische Wochenschrift," Leipzig, December. 1891.

(11) De la Sota, "El Liglio," Madrid, Jannary, 1897.

112) Compaired, "Revista de Laringologia," Barcelona.

1 13) Felix Semon, "British Medical Journal," January 5th, 1895.

(14) Wm. C. Glasgow, "Burnett's Sistem of Diseases of the Throat."

115) Bayer, "Journal of Laryngology," vol. ix.

116) Castex, "Revue de Laryngologie," 1896.

117) John M. Mackenzie, "Johns Hopkins Bulletin," 1898. 